ESJ Social Sciences

\title{
Actividades y Funciones Institucionales de Profesores en una Escuela de Negocios en el Centro de México
}

\author{
Barajas Villarruel Juan Ignacio \\ Universidad Autónoma de San Luis Potosí, México
}

Doi:10.19044/esj.2021.v17n5p232

Submitted: 25 January 2021

Accepted: 27 February 2021

Published: 28 February 2021
Copyright 2021 Author(s)

Under Creative Commons BY-NC-ND

4.0 OPEN ACCESS

Cite As:

Juan Ignacio B.V. (2021). Actividades y Funciones Institucionales de Profesores en una Escuela de Negocios en el Centro de México. European Scientific Journal, ESJ, 17(5), 232. https://doi.org/10.19044/esj.2021.v17n5p232

\section{Resumen}

El objetivo de la investigación fue determinar la distribución de actividades y funciones institucionales de los profesores de tiempo completo, técnicos académicos y profesores de asignatura de la Facultad de Contaduría y Administración de una universidad pública del centro de México. La investigación fue no experimental, descriptiva y transversal. Se aplicó el cuestionario Diagnóstico de Actividades y Funciones Institucionales de Profesores de la FCA con preguntas cerradas que se midieron con una escala de Likert, para determinar la distribución de las actividades de investigación, docencia, gestión y tutoría, de los profesores participantes. El tipo de muestra fue no probabilístico del tipo causal o incidental conformada por 23 profesores. El principal resultado fue que las actividades y funciones de los profesores participantes del estudio no están equilibradas, tomando como base sobre las disposiciones de los reglamentos institucionales, así como, de los organismos externos a los que se hace referencia en el estudio.

Palabras clave: Diagnóstico, Profesor, Actividades y/o Funciones Institucionales, Escuelas de Nivel superior 


\title{
Activities and Institutional Functions of Professors of a Business School in Central Mexico
}

\author{
Barajas Villarruel Juan Ignacio \\ Universidad Autónoma de San Luis Potosí, México
}

\begin{abstract}
The objective of the research was to determine the distribution of activities and institutional functions of full-time professors, academic technicians and time partial professor of the Faculty of Accounting and Administration of a public university in central Mexico. The research was nonexperimental, descriptive, and transversal. The FCA Professors' Institutional Activities and Functions Diagnostic questionnaire was applied with closed questions that were measured with a Likert scale, to determine the distribution of research, teaching, management and tutoring activities of the participating teachers. The population sample was non-probabilistic and using an incidental type, conformed 23 teachers. The main result was that the activities and functions of the participating the study are not balanced, based on the provisions of the institutional regulations and the external bodies referenced in the study.
\end{abstract}

Keywords: Diagnostic, Professor, Institutional Activities and/or Functions, Higher Level School

\section{Introduction}

El siglo que vivimos se caracteriza por una invariante: el cambio. Este se evidencia tanto en lo social, político, económico, así como en la tecnología, la educación y el trabajo. Según Palomares (2004), el siglo XXI presenta los rasgos siguientes: la globalización como un proceso generalizado, el papel de los medios masivos como promotores de nuevos modos de vida y consumo, la flexibilización de la figura de autoridad, la información oportuna como insumo para la toma de decisiones entorno a lo económico y político, el avance constante de la tecnologías de información y comunicación, y el individualismo como modelo de vida, en síntesis según el autor, la migración hacia una sociedad del conocimiento.

En este contexto, las universidades están inmersas en una profunda trasformación de su identidad. Este cambio va hacia la construcción de una nueva estructura que permita la apropiación del conocimiento por sectores de la sociedad que antes no contaban con esta posibilidad y a partir de esto la formación de individuos que atiendan los desafíos de la sociedad actual (Goñi, 2005). Asimismo, como afirmó Tomàs (2001), es necesario analizar la organización de la universidad dejando a un lado el modelo burocrático y 
encaminarse a la flexibilidad de los aspectos tanto como curriculares así como, de la redefinición del rol de los estudiantes, de los procesos educativos y de investigación y, para el caso de este trabajo, el rol de los profesores.

A este respecto, los profesores universitarios, también en su calidad de investigadores, están involucrados en funciones de docencia así como, del fomento a la investigación competitiva y formación de grupos de trabajo multidisciplinarios (Ruiz, Mas, Tejada \& Navío,2008). Otras actividades asignadas a los profesores de este nivel educativo son la de gestión y tutorías. Para el caso de la Universidad Autónoma de San Luis Potosí, institución a la que pertenece la sede de esta investigación, según Reglamento del Personal Académico en el artículo dos, el profesor:

[...] tiene a su cargo la impartición de la enseñanza universitaria en todos sus niveles, de los planes de estudio y programas aprobados, la realización de la investigación y la difusión de la cultura, de acuerdo con el contenido de los planes de estudio y programas aprobados (UASLP, 1984, p.1).

Además, según este reglamento, todas las actividades realizadas por los profesores se llevarán al cabo bajo el principio de libre cátedra, entendida como plena libertad para la exposición y discusión de ideas, en el marco de sus responsabilidades académicas y administrativas (UASLP, 1984).

Adicionalmente, la normativa de esta institución reconoce como personal académico al profesor o investigador, técnico académico y profesores de asignatura. Los profesores e investigadores de carrera son quienes destinan formalmente su carga laboral en labores académicas. Por otro lado, los técnicos académicos son quienes a partir de su experiencia profesional destinan su carga laboral a realizar tareas específicas y sistemáticas del apoyo a los programas académicos o de otras actividades técnicas de investigación. Finalmente, son profesores de asignatura los que imparten docencia por determinado número de horas-clase (UASLP, 1984).

Un referente que orienta y evalúa las actividades de investigación de los profesores universitarios es el Sistema Nacional de Investigadores (SNI) del Consejo Nacional de Ciencia y Tecnología (CONACYT). Este sistema fue creado el 26 de julio de 1984, con el propósito de reconocer el trabajo de las personas dedicadas a producir conocimiento científico y tecnológico, a través de otorgar uno de los cuatro nombramientos de investigador nacional (Candidato, Nivel 1, Nivel 2 o Nivel 3), así como estímulos económicos cuyo monto varía con el nivel asignado (SNI, 2014).

Al respecto, en las convocatorias anuales de selección del SNI los profesores deben comprobar que realizan habitual y sistemáticamente actividades de investigación científica o tecnológica, así como, presentar los productos del trabajo debidamente documentados en un sistema de 
información en línea. Asimismo, para el caso de los profesores de la facultad sede de este diagnóstico, deben cumplir con los requisitos de estar contratados por la UASLP con un nombramiento PTC o TA y demostrar, por medio de documento oficial original y actualizado, que presta servicios por al menos 20 horas a la semana para realizar actividades de investigación científica o tecnológica (CONACYT, 2019). Lo anterior obliga a los aspirantes a ingresar al SNI, la dedicación de al menos 20 horas a la semana al desarrollo de la función de investigación, sin considerar las funciones de Docencia, Gestión y Tutorías que se deben distribuir en las 20 horas a la semana restantes de su carga laboral.

Por otra parte, también existe el referente del Programa para el Desarrollo Profesional Docente Para el Tipo Superior (PRODEP) que orienta y promueve la mejor actuación profesional de los profesores universitarios. Este programa está dirigido a elevar permanentemente el nivel de habilitación del profesorado, con base en los perfiles adecuados para cada subsistema de educación superior. Este programa tiene como propósito elevar la calidad de la educación superior a través de la superación permanente en los procesos de formación, dedicación y desempeño de los profesores y cuerpos académicos de las instituciones educativas de nivel superior (SEP, 2019).

Para lograr el propósito del PRODEP se estableció como uno de sus principales objetivos el fortalecer los procesos de habilitación y mejoramiento de los Profesores de Tiempo Completo (SEP, 2019). Para dicho objetivo, el PRODEP estableció las siguientes estrategias: la contratación y habilitación de los PTC, la creación y fortalecimiento de Cuerpos Académicos. Las principales acciones que el PRODEP establece para lograr sus objetivos son (SEP, 2019):

1. Otorgar becas nacionales y para el extranjero a profesores de carrera de las universidades públicas, para la realización de estudios de posgrado en programas de reconocida calidad.

2. Apoyar la contratación de nuevos profesores de tiempo completo que ostenten el grado académico de maestría o de doctorado (preferentemente) y la reincorporación de exbecarios PRODEP (profesores beneficiados con una beca para estudios de posgrado).

3. Reconocer con el Perfil Deseable a profesores que cumplen, con eficacia y equilibrio sus funciones (docencia, investigación, gestión, tutorías) de profesor de tiempo completo.

4. Apoyar el fortalecimiento de Cuerpos Académicos y la integración de redes temáticas de colaboración.

Específicamente, para acceder al reconocimiento de Perfil Deseable y/o participar como miembro o colaborador de un Cuerpo Académico, el PRODEP solicita que los aspirantes capturen en un sistema de información en 
línea, la información y documentos que comprueben que existe un equilibrio de su carga laboral entre las actividades y funciones institucionales de Investigación, Docencia, Gestión y Tutorías. Esta es una de las razones por la cual es pertinente la elaboración de esta evaluación diagnóstica.

\section{Problema de Estudio:}

La Facultad de Contaduría y Administración (FCA), sede de la presente investigación es una dependencia universitaria pública con oferta educativa a nivel pregrado y posgrado. En el caso del nivel pregrado existen programas de licenciatura en Contaduría Pública, Administración, Administración Pública, Agronegocios y Mercadotecnia Estratégica. El nivel de Posgrado de la facultad cuenta con los programas de Maestría en Administración con énfasis en Negocios, Gestión Pública e Impuestos; y Maestría en Alta Dirección en conjunto con la Universidad de Québec en Montréal Canadá y el programa de Doctorado en Ciencias Administrativas. Asimismo, la matrícula de esta institución es de 3698 estudiantes de licenciatura y 209 de posgrado, todos ellos atendidos por 279 profesores. (UASLP, 2019)

Por otra parte, para el caso del personal docente, una de las formas de organización académica, de acuerdo con las políticas de educación superior, es la de Cuerpos Académicos (PRODEP, 2019). Como una iniciativa para generar una temática de investigación, se problematizó la cuestión de conocer cuáles son las actividades y funciones institucionales que los profesores realizan en su carga laboral. En consecuencia, se planteó el siguiente problema de investigación: se desconoce la distribución de actividades y funciones institucionales de los Profesores de Tiempo Completo, Técnicos Académicos y Profesores de asignatura de la FCA.

\section{Objetivo General:}

Determinar la distribución de actividades y funciones institucionales de los Profesores de Tiempo Completo, Técnicos Académicos y Profesores de Asignatura de la Facultad de Contaduría y Administración de una universidad pública del centro de México.

Lo anterior para: (a) identificar áreas de oportunidad que permitan equilibrar las actividades y funciones de Investigación, Docencia, Gestión y Tutorías del personal académico, (b) asegurar su adecuada participación en las diferentes convocatorias del Programa para el Desarrollo Profesional Docente para el Tipo Superior (PRODEP), las del Consejo Nacional de Ciencia y Tecnología (CONACYT), principalmente la del Sistema Nacional de Investigadores (SNI) y las de la misma universidad, como por ejemplo la de la Beca al desempeño Docente; y finalmente (c) coadyuvar al logro de 
indicadores de investigación, docencia, gestión y tutorías establecidos en las políticas y programas del Plan Institucional de Desarrollo 2013-2023.

\section{Preguntas de Investigación:}

El presente estudio se centra en las siguientes preguntas de investigación:

1. ¿Cuál es la distribución de actividades y funciones institucionales de los Profesores de Tiempo Completo de la Facultad de Contaduría y Administración de la FCA de una universidad pública del centro de México?

2. ¿Cuál es la distribución de actividades y funciones institucionales de los Técnicos Académicos de la Facultad de Contaduría y Administración de la FCA de una universidad pública del centro de México?

3. ¿Cuál es la distribución de actividades y funciones institucionales de los Profesores de asignatura de la Facultad de Contaduría y Administración de la FCA de una universidad pública del centro de México?

\section{Metodología:}

\subsection{Diseño del estudio}

El presente trabajo fue un estudio de tipo no experimental en el que se aplicó la técnica de investigación de encuesta. No se construyó situación alguna, se analizó cuantitativamente la distribución de las de actividades y funciones institucionales de investigación, docencia, gestión y tutorías, dentro de la carga laboral de los profesores participantes.

\subsection{Muestra}

El tipo de muestra fue no probabilístico del tipo causal o incidental. Según Hernández et al. (2014), este tipo de muestras no obedecen a la probabilidad, si no a causas relacionadas con las características de la investigación. Este tipo de muestreo faculta al investigador a convocar de una población a participar de manera voluntaria en el estudio. En consecuencia, se recupera información solo de los voluntarios que atienden la convocatoria.

Finalmente, la muestra se conformó a partir de una invitación a la población de profesores de FCA que actualmente cuentan con un nombramiento de PTC, TA y PHC. Los voluntarios participantes fueron 23 profesores, 17 de ellos con nombramiento de PTC, un TA y cinco PHC. La Tabla 1 indica que del total de participantes, 18 son miembros o colaboradores de los cinco Cuerpos Académicos de la facultad registrados ante el PRODEP (SEP, 2019). 
Tabla 1. Miembros o colaboradores de Cuerpos Académicos que participaron en el diagnóstico.

\begin{tabular}{|c|c|c|c|c|}
\hline No. & Nombre del CA & Grado & $\begin{array}{c}\text { Total de } \\
\text { miembros o } \\
\text { colaboradores del } \\
\text { CA }\end{array}$ & $\begin{array}{c}\text { Total de } \\
\text { participantes } \\
\text { en el } \\
\text { diagnóstico. }\end{array}$ \\
\hline 1 & $\begin{array}{l}\text { UASLP-CA-212 } \\
\text { Innovación y Gestión } \\
\text { en las Organizaciones }\end{array}$ & Consolidado & $8(100 \%)$ & $5(62.5 \%)$ \\
\hline 2 & $\begin{array}{l}\text { UASLP-CA-204 } \\
\text { Innovación, } \\
\text { Administración y } \\
\text { Gestión de Procesos } \\
\text { Educativos para las } \\
\text { Organizaciones }\end{array}$ & En Consolidación & $6(100 \%)$ & $4(66.66 \%)$ \\
\hline 2 & $\begin{array}{l}\text { UASLP-CA-205 } \\
\text { Estudios } \\
\text { Organizacionales } \\
\text { UASLP-CA-230 }\end{array}$ & En Formación & $6(100 \%)$ & $4(66.66 \%)$ \\
\hline 4 & $\begin{array}{l}\text { Nuevas Tendencias en } \\
\text { Información Financiera } \\
\text { para la Gestión }\end{array}$ & En Formación & $4(100 \%)$ & $2(50 \%)$ \\
\hline 5 & $\begin{array}{l}\text { UASLP-CA-255 } \\
\text { Desarrollo innovador y } \\
\text { competitivo de las } \\
\text { organizaciones }\end{array}$ & En Formación & $6(100 \%)$ & $3(50 \%)$ \\
\hline
\end{tabular}

Fuente: PRODEP (2019)

\subsection{Instrumento}

Para determinar la distribución de funciones profesionales de los profesores de la FCA se diseñó y validó por expertos el cuestionario denominado "Diagnóstico de Actividades y Funciones Institucionales de Profesores de la FCA". El cuestionario contiene lo siguiente: objetivo del cuestionario, instrucciones de llenado y dos secciones con 38 ítems. La primera sección es para determinar el perfil académico de los participantes y la segunda sección está distribuida en las dimensiones de Investigación, Docencia, Gestión y Tutorías.

\subsection{Validación del instrumento}

Se realizaron dos tipos de validación de contenido y prueba piloto. La validación de contenido del cuestionario estuvo basada en el juicio de tres expertos en diseño de instrumentos de investigación y la prueba piloto consistió en aplicar el cuestionario a cinco profesores de otras facultades de la universidad sede del estudio.

Los resultados de la validación de contenido por parte de expertos recomendaron cambios de forma, referidos a la redacción de las preguntas. 
También, indicaron eliminar signos de interrogación y recomendaron la reestructuración de la redacción del objetivo del cuestionario.

Para la prueba piloto, se solicitó responder el cuestionario a cinco profesores que no pertenecieron a la muestra. Los cuestionarios de esta prueba piloto incluyeron tres columnas para indicar los siguientes aspectos: (a) cuáles ítems son imprecisos, (b) cuáles ítems no están relacionados el propósito de la investigación, y (c) cuáles ítems sugiere incluir en el cuestionario. Los resultados de la prueba recomendaron incluir dos nuevos ítems para la dimensión de Gestión. También es importante señalar que no se eliminó o modifico ningún otro ítem.

\section{Resultados:}

Los resultados se presentan con relación a los ítems del instrumento denominado "Diagnóstico de Actividades y Funciones Institucionales de Profesores de la FCA". En primer lugar, se presentan los datos del perfil general de los participantes. En segundo lugar, los resultados de actividades y funciones institucionales de los profesores participantes.

\subsection{Datos del Perfil General de los Participantes}

El total de participantes fueron 23 personas. El 52.2\% del total son profesores con doctorado, el $39.1 \%$ con maestría, $4.3 \%$ con postdoctorado y 4.3\% con licenciatura. La Figura 1 muestra la distribución de frecuencias del grado académico que tienen los participantes del diagnóstico.

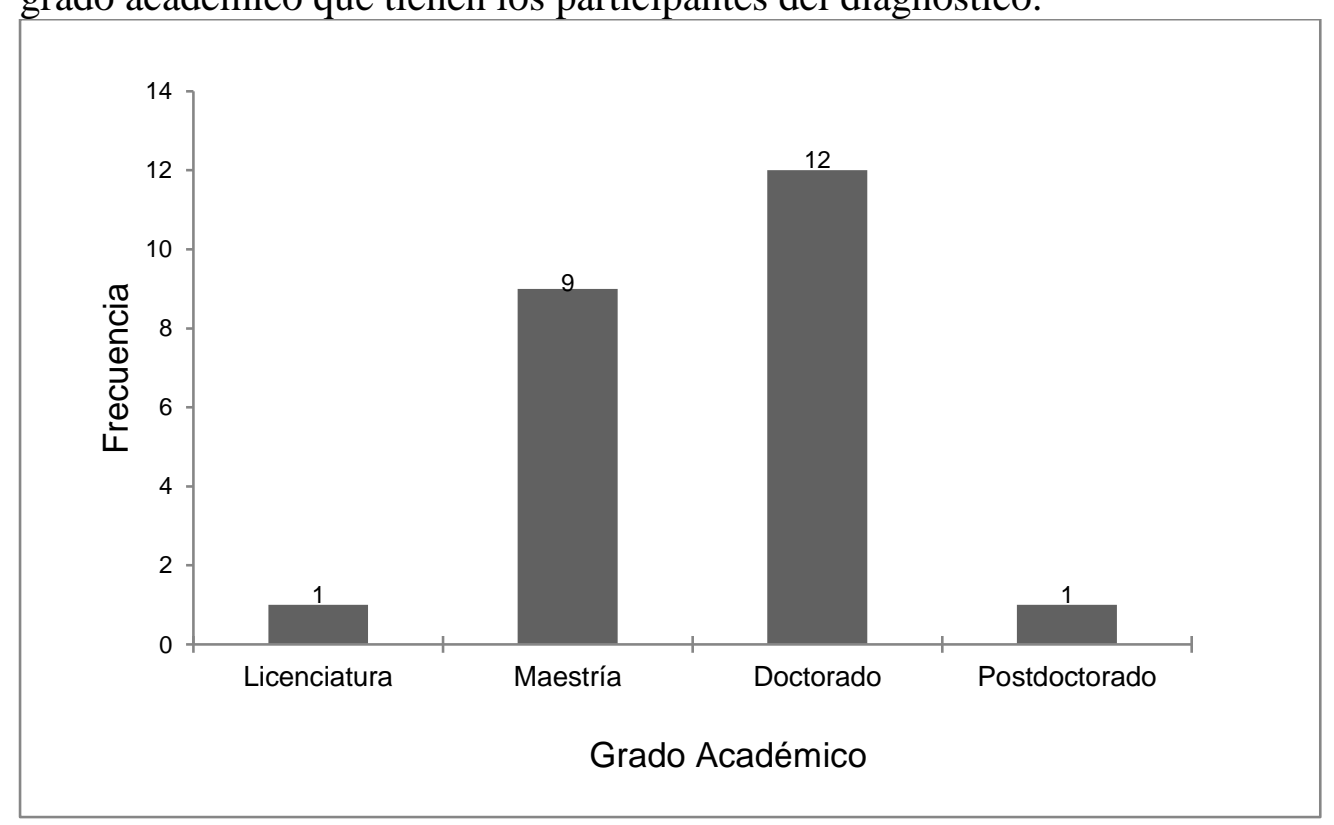

Figura 1. Distribución de frecuencias de Grados Académicos. 
Por otro lado, La Figura 2 representa los nombramientos institucionales que tienen los profesores participantes. El $73.9 \%$ de los profesores cuentan con nombramiento de Profesor de Tiempo Completo (PTC), el $21.7 \%$ con nombramiento de Profesor Hora Clase (PHC) y el $4.3 \%$ con un nombramiento de Técnico Académico (TA). De los cinco PHC, tres de ellos informaron que son categoría tres, uno categoría dos y uno más categoría uno. Igualmente, el profesor con nombramiento de TA reportó contar con el nivel dos del tabulador definido para TA. La Figura 2 representa los nombramientos institucionales que tienen todos los profesores participantes.

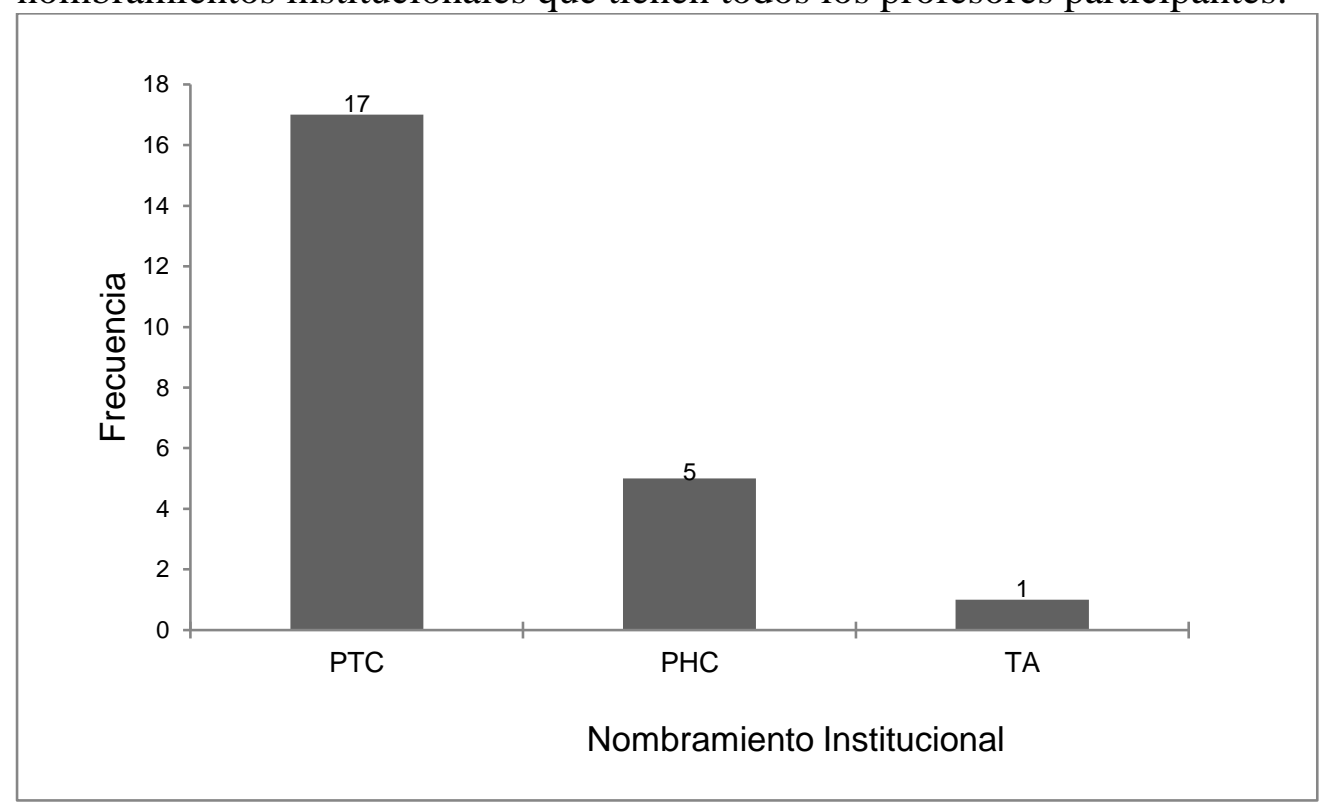

Figura 2. Distribución de Nombramientos Institucionales.

Del total de PTC se reportó que 16 de ellos son Nivel seis y uno el Nivel cinco. También, es importante señalar que 16 de los 17 PTC actualmente cuentan con reconocimiento por tres años de Perfil Deseable que otorga el Programa para el Desarrollo Docente (PRODEP). Con respecto al Sistema Nacional de Investigadores (SNI), los PTC reportaron que siete de ellos pertenecen a dicho sistema, cuatro con Nivel uno y tres son Candidatos.

\subsection{Actividades y Funciones Institucionales de los Profesores Participantes}

Para presentar estos resultados se consideró el orden de las actividades y funciones indicadas en el diagnóstico, investigación, docencia, gestión y tutoría. 


\subsubsection{Investigación}

Una de las principales obligaciones de los PTC es realizar investigación científica con parámetros mínimos de calidad establecidos por la UASLP, organismos nacionales (PRODEP, CONACYT, etc.) e internacionales (revistas indexadas consideradas en Journal Citation Report y otras editoriales de calidad a nivel mundial). Al respecto Figueiredo, Montoro \& Lucena (2016), resaltaron que la actividad de investigación además coadyuba a un cambio positivo en la actividad profesional aumentado su responsabilidad y compromiso en la labor que desempeñan.

Los indicadores considerados en la función de Investigación Científica son: (a) horas semanales (Hrs/Sem), (b) número de proyectos de investigación en proceso, (c) financiamiento interno o externo, (d) número de tesis en las que participa como director o asesor de tesis de licenciatura y posgrado con o sin reconocimiento del Programa Nacional de Posgrados de Calidad (PNPC), (e) número de ocasiones en las que ha recibido financiamiento por parte de las autoridades de la facultad para la difusión de resultados en congresos nacionales y/o internacionales y (f) participación en Cuerpos Académicos.

Horas/Semanales dedicadas a proyectos de investigación. Para realizar la función de Docencia es necesario, de acuerdo con el PRODEP y CONACYT, que los PTC dediquen un mínimo de 20 horas/semana de su carga de 40 horas/semana. Si bien es cierto, esta función es inherente a los PTC y TA, también es cierto que los PHC pueden participar formalmente como colaboradores de proyectos de investigación, figura reconocida por el PRODEP. La Figura 3 contiene el número de horas/semana que los PTC y TA dedican para realizar investigación científica.

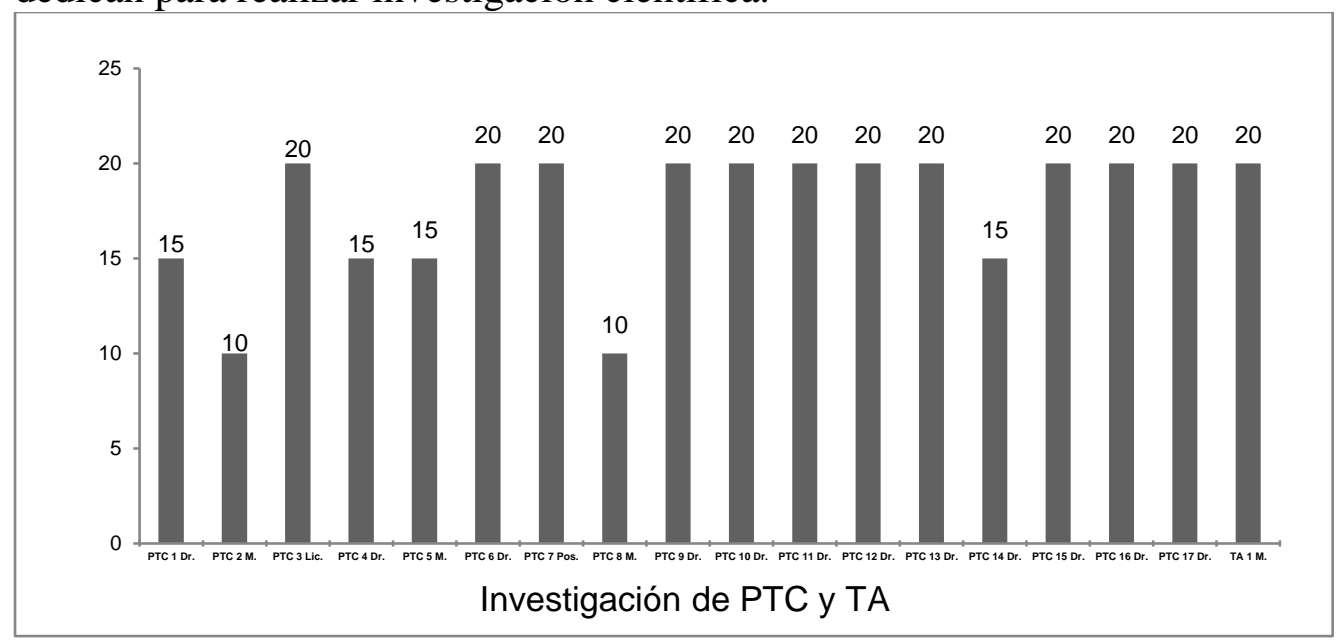

Figura 3. Hrs/Sem que dedican a la investigación los PTC y TA. 
También, tres de los cinco PHC reportaron realizar o colaborar con PTC en proyectos de investigación. La Figura 4 contiene el número de horas/semana que los PHC dedican a la función de investigación.

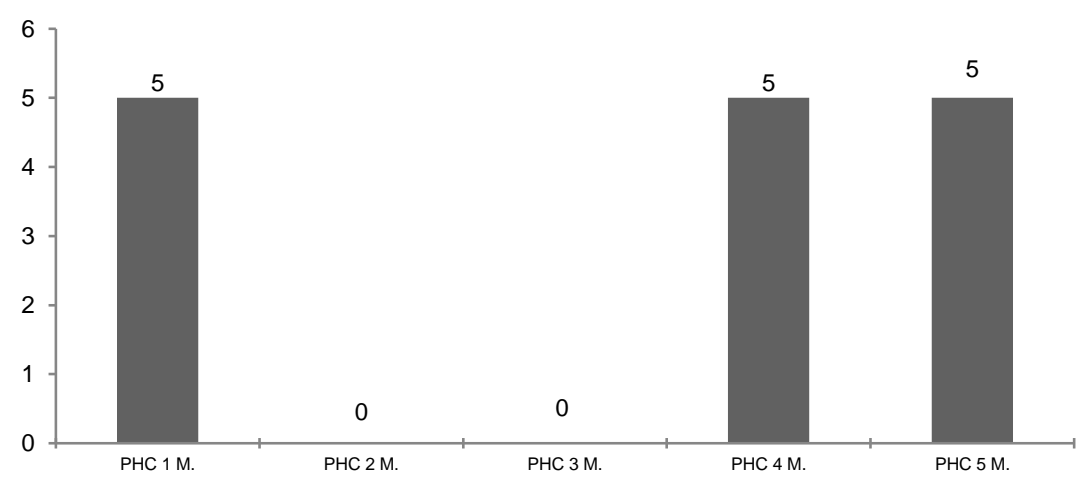

Investigación de Profesores Asignatura u Hora Clase

Figura 4. Hrs/Sem de investigación que dedican los Profesores Asignatura u Hora Clase.

Proyectos de investigación en proceso. Para este indicador, los profesores participantes reportaron el número de proyectos en los que actualmente están trabajando. La Figura 5 presenta el número de proyectos en los que actualmente están trabajando los PTC y TA. Cabe señalar que todos los PTC y TA reportan estar trabajando en al menos un proyecto de investigación, mientras que de los cinco PHC solo uno de ellos reportó que está colaborando en dos proyectos de investigación.

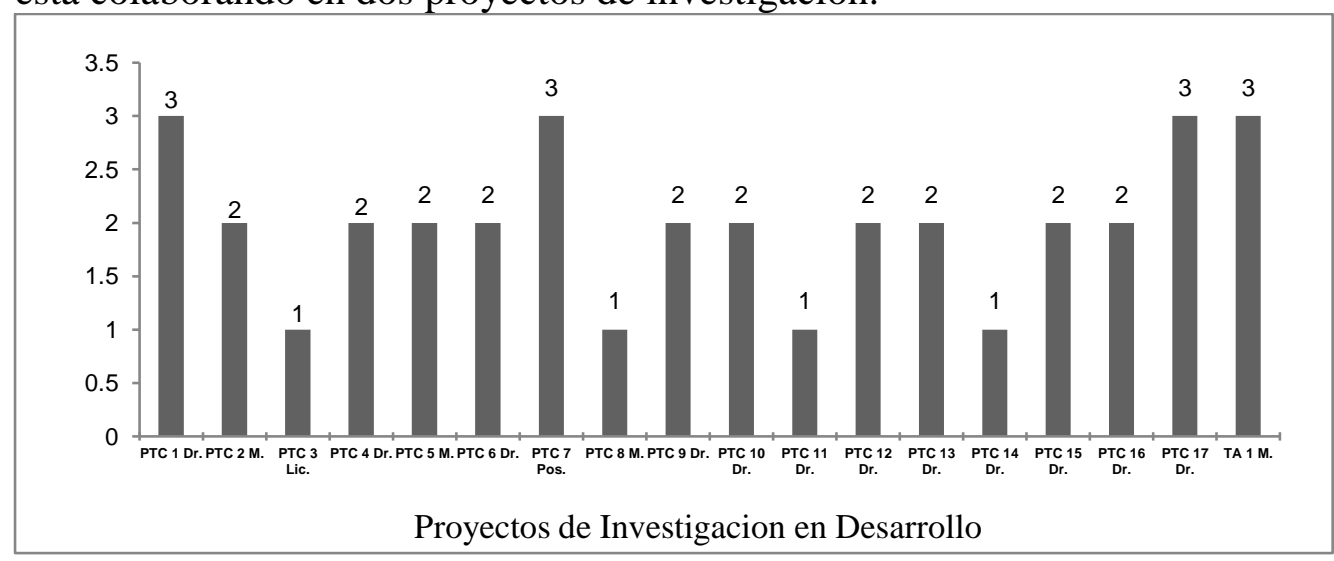

Figura 5. No. de proyectos de investigación en desarrollo.

Financiamiento para proyectos de investigación. Los resultados establecieron que solo dos PTC, de los 17 que participaron en el estudio, recibieron en el último año algún apoyo financiero institucional para el desarrollo de sus proyectos de investigación. Por otro lado, no se reportó 
ningún apoyo financiero por parte de las autoridades de la FCA para proyectos en los que participan TA o PHC.

Para el caso del financiamiento externo para el desarrollo de proyectos de investigación, solo siete PTC, de todos los profesores que participaron en el diagnóstico, reportaron que en el último año sí han recibido financiamiento para al menos uno de sus proyectos de investigación (PRODEP, CONACYT, etc.). A continuación, la Tabla 2 contiene información sobre apoyo financiero interno y/o externo para el desarrollo de sus investigaciones y si ellos pertenecen a un Cuerpo Académico de la FCA registrado en el PRODEP (SEP, 2019).

Tabla 2. Financiamiento interno y/o externo para proyectos de investigación

\begin{tabular}{|c|c|c|c|}
\hline $\begin{array}{l}\text { Participante del } \\
\text { estudio }\end{array}$ & $\begin{array}{l}\text { Pertenece a un CA de la } \\
\text { FCA/Grado de CA }\end{array}$ & $\begin{array}{l}\text { Financiamiento } \\
\text { Interno }\end{array}$ & $\begin{array}{c}\text { Financiamiento } \\
\text { Externo }\end{array}$ \\
\hline PTC1 Dr & Si / En Formación & No & $\mathrm{Si}$ \\
\hline PTC2 M. & Si / En Formación & No & No \\
\hline PTC3 Lic. & Si / En Consolidación & No & No \\
\hline PTC4 Dr & Si / En Consolidación & No & No \\
\hline PTC5 M. & Si / En Formación & No & No \\
\hline PTC6 Dr & Si / En Formación & No & $\mathrm{Si}$ \\
\hline PTC7 Pos. & Si / Consolidado & No & No \\
\hline PTC8 M. & Si / En Formación & No & No \\
\hline PTC9 Dr & $\mathrm{Si} /$ Consolidado & $\mathrm{Si}$ & $\mathrm{Si}$ \\
\hline PTC10 Dr & Si / En Formación & No & No \\
\hline PTC11 Dr & Si / En Formación & No & $\mathrm{Si}$ \\
\hline PTC12 Dr & Si / Consolidado & No & No \\
\hline PTC13 Dr & Si / En Formación & No & $\mathrm{Si}$ \\
\hline PTC14 Dr & Si / En Formación & No & No \\
\hline PTC15 Dr & Si / En Formación & $\mathrm{Si}$ & $\mathrm{Si}$ \\
\hline PTC16 Dr & Si / Consolidado & No & $\mathrm{Si}$ \\
\hline PTC17 Dr & Si / En Consolidación & No & No \\
\hline TA M. & Si / En Consolidación & No & No \\
\hline PHC1 M. & No & No & No \\
\hline PHC2 M. & No & No & No \\
\hline PHC3 M. & No & No & No \\
\hline PHC4 M. & No & No & No \\
\hline PHC5 M. & No & No & No \\
\hline
\end{tabular}

Participación como director o asesor de tesis de licenciatura y posgrado con o sin PNPC. Uno de los principales aspectos considerados en la función de investigación es la formación de nuevos investigadores a través del desarrollo de proyectos de investigación y de la dirección o asesoría de tesis en nivel de licenciatura, maestría o doctorado. Al respecto, la Tabla 3 muestra la cantidad de tesis en las que actualmente son directores de tesis o en las que el último año han tenido el rol de asesores, todas estas en los diferentes niveles académicos. 
Tabla 3. No. de ocasiones en las que en el último año participaron como director y/o asesor de Tesis.

\begin{tabular}{lcccccccc}
\hline & \multirow{2}{*}{ Licenciatura } & \multicolumn{2}{c}{$\begin{array}{c}\text { Posgrado sin } \\
\text { PNPC }\end{array}$} & \multicolumn{2}{c}{ Posgrado con } & \multicolumn{2}{c}{ Total } \\
Participante & \multicolumn{2}{c}{} & \multicolumn{2}{c}{ PNC } & Asesor \\
\cline { 2 - 9 } & Director & Asesor & Director & Asesor & Director & Asesor & Director & A \\
& 2 & 2 & 5 & 6 & 0 & 0 & 7 & 8 \\
\hline PTC1 Dr & 2 & 0 & 2 & 0 & 0 & 0 & 4 & 0 \\
PTC2 M. & 0 & 0 & 0 & 0 & 0 & 0 & 0 & 0 \\
PTC3 Lic. & 0 & 0 & 0 & 0 & 0 & 0 & 0 & 0 \\
PTC4 Dr & 0 & 1 & 0 & 0 & 0 & 0 & 0 & 1 \\
PTC5 M. & 0 & 0 & 5 & 3 & 0 & 0 & 5 & 3 \\
PTC6 Dr & 0 & 2 & 3 & 4 & 2 & 2 & 6 & 8 \\
PTC7 Pos. & 1 & 0 & 0 & 0 & 0 & 0 & 0 & 0 \\
PTC8 M. & 0 & 0 & 0 & 4 & 1 & 0 & 3 & 4 \\
PTC9 Dr & 2 & 0 & 4 & 2 & 0 & 0 & 4 & 2 \\
PTC10 Dr & 0 & 0 & 0 & 1 & 0 & 0 & 6 & 1 \\
PTC11 Dr & 0 & 0 & 6 & 0 & 0 & 0 & 0 & 2 \\
PTC12 Dr & 0 & 2 & 0 & 0 & 0 & 0 & 4 & 1 \\
PTC13 Dr & 0 & 1 & 4 & 0 & 0 & 0 & 2 & 0 \\
PTC14 Dr & 0 & 0 & 2 & 0 & 0 & 4 & 2 \\
PTC15 Dr & 0 & 0 & 4 & 2 & 0 & 0 & 5 & 5 \\
PTC16 Dr & 0 & 2 & 3 & 3 & 2 & 0 & 1 & 0 \\
PTC17 Dr & 0 & 0 & 0 & 0 & 1 & 0 & 0 & 0 \\
TA M. & 0 & 0 & 0 & 0 & 0 & 0 & 0 & 0 \\
PHC1 M. & 0 & 0 & 0 & 0 & 0 & 0 & 0 & 0 \\
PHC2 M. & 0 & 0 & 0 & 0 & 0 & 0 & 0 & 0 \\
PHC3 M. & 0 & 0 & 0 & 0 & 0 & 0 & 0 & 0 \\
PHC4 M. & 0 & 0 & 0 & 0 & 0 & 0 & 0 & 0 \\
PHC5 M. & 0 & 0 & 0 & 0 & 0 & 0 & 0 & 0 \\
\hline
\end{tabular}

Difusión de resultados. Otro indicador para considerar es la difusión de resultados que implica la participación en congresos reconocidos a nivel nacional e internacional, así como, la publicación de los mismos en revistas arbitradas e indexadas. Para lo anterior es necesario contar con el financiamiento, para el caso de congresos, de transportes, hospedaje, inscripción, alimentación, y para algunas revistas indexadas el costo de publicación.

La Tabla 4 presenta la frecuencia de apoyos a congresos nacionales o internacionales que se han otorgado el último año a los profesores que participaron en el estudio por parte de las autoridades de la FCA. 
Tabla 4. No. de ocasiones en las que en el último año recibieron financiamiento de las

\begin{tabular}{lcccc}
\multicolumn{4}{c}{ autoridades de la FCA para la difusión de resultados. } \\
\cline { 2 - 4 } Participante & Locales & Nacional & Internacional & \\
\cline { 2 - 4 } & 0 & 1 & 1 & 2 \\
\hline PTC1 Dr & 0 & 0 & 0 & 0 \\
PTC2 M. & 0 & 0 & 0 & 0 \\
PTC3 Lic. & 0 & 0 & 0 & 0 \\
PTC4 Dr & 0 & 0 & 0 & 0 \\
PTC5 M. & 0 & 0 & 0 & 0 \\
PTC6 Dr & 1 & 1 & 1 & 3 \\
PTC7 Pos. & 0 & 0 & 0 & 0 \\
PTC8 M. & 0 & 0 & 0 & 0 \\
PTC9 Dr & 0 & 0 & 0 & 0 \\
PTC10 Dr & 0 & 0 & 0 & 0 \\
PTC11 Dr & 0 & 1 & 1 & 2 \\
PTC12 Dr & 0 & 1 & 0 & 1 \\
PTC13 Dr & 0 & 0 & 0 & 0 \\
PTC14 Dr & 0 & 2 & 0 & 2 \\
PTC15 Dr & 0 & 0 & 1 & 1 \\
PTC16 Dr & 0 & 0 & 0 & 0 \\
PTC17 Dr & 0 & 0 & 0 & 0 \\
TA M. & 0 & 0 & 0 & 0 \\
PHC1 M. & 0 & 0 & 0 & 0 \\
PHC2 M. & 0 & 0 & 0 & 0 \\
PHC3 M. & 0 & 0 & 0 & 0 \\
PHC4 M. & 0 & 1 & 0 & 1 \\
PHC5 M. & 0 & & & \\
\hline
\end{tabular}

\subsubsection{Docencia}

Otra función de todos los PTC y PHC es impartir docencia en los niveles de licenciatura y/o posgrado. Los principales criterios que se toman como referencia para impartir cátedra en la universidad son: contar con el perfil profesional afín a la naturaleza del curso que se oferta, antigüedad institucional y que al menos el profesor cuente con el mismo nivel académico del curso que va a impartir (UASLP; 1984).

Para determinar la carga de Hrs/Sem de cada uno de los participantes, se les cuestionó el número de horas que tienen frente a grupo a la semana y el nivel en el que las imparte, licenciatura y/o posgrado registrado o no en el PNPC del CONACYT (Ver Figura 6). Para el caso de los PHC que participaron en el estudio reportaron que toda su carga docente es a nivel licenciatura. 


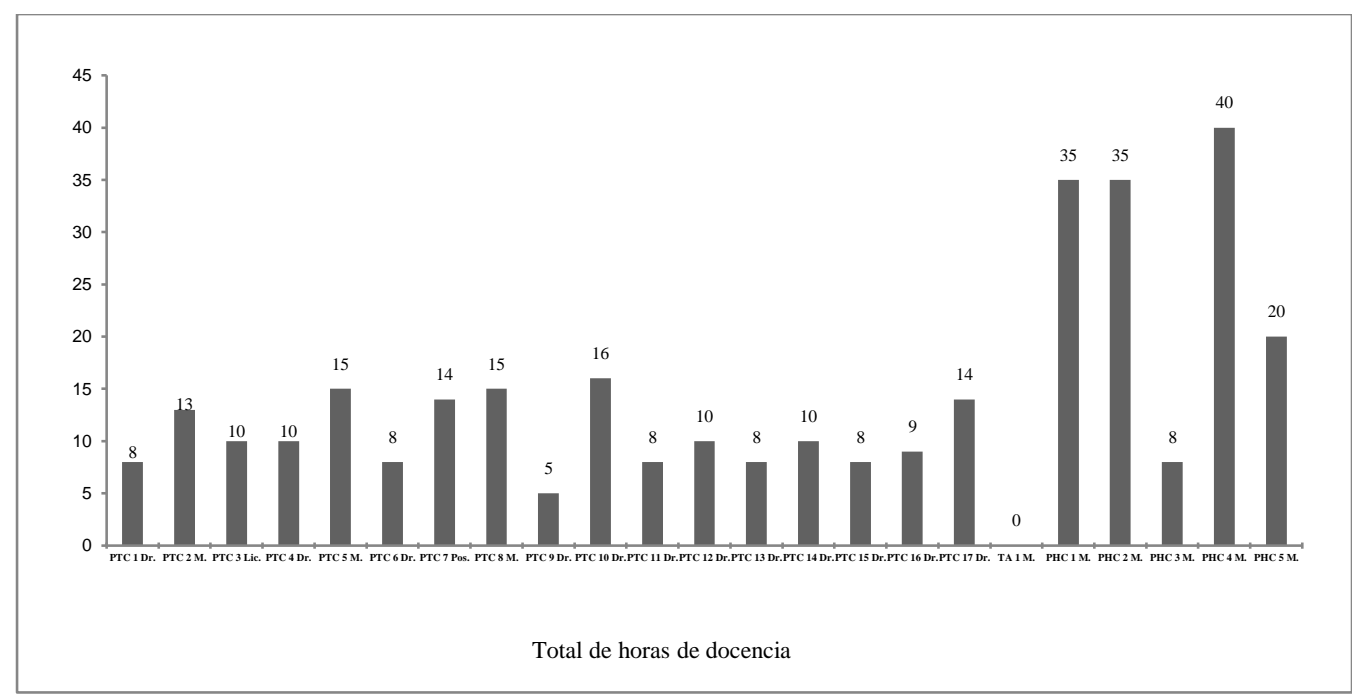

Figura 6. Hrs/Sem de docencia que dedican los Profesores.

La Figura 7, presenta la distribución de la Docencia de los PTC y TA en los niveles de licenciatura y/o posgrado registrado o no en el PNPC del CONACYT.

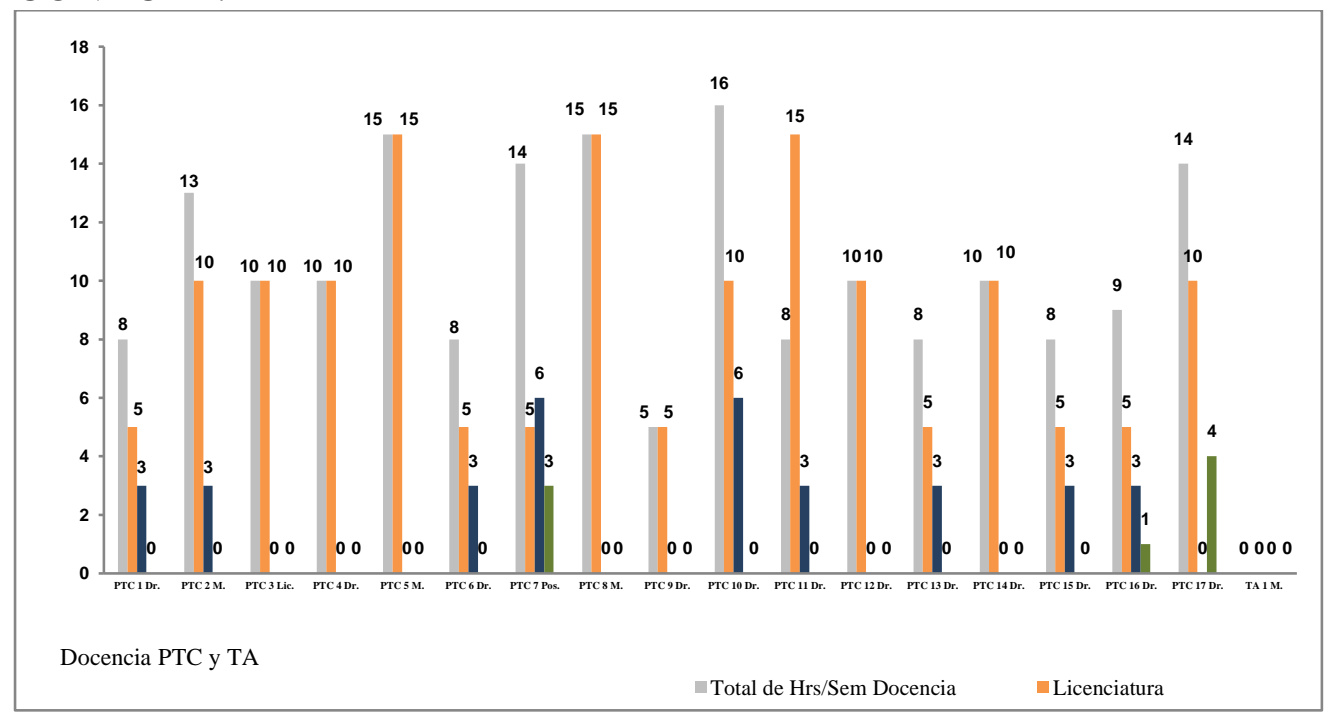

Figura 7. Total de Hrs/Sem que dedican los PTC y TA a la Docencia en los diferentes niveles académicos

\subsubsection{Gestión}

Los resultados de esta función consideraron la participación de los profesores en actividades de diseño/rediseño del Modelo educativo de la facultad, así como, la participación en la definición/redefinición de programas sintéticos y/o analíticos. Asimismo, se cuestionó si en el último año han tenido a su cargo alguna responsabilidad institucional (jefaturas, coordinaciones, 
Consejo Técnico de la Facultad, H. Consejo Universitario, etc.) y/o alguna función como responsables/corresponsables en eventos académicos concretos (examen de admisión, Verano de la Ciencia, Semana del FCA, Semana Nacional de Ciencia y Tecnología, etc.) La Tabla 5 presenta la frecuencia de las actividades de gestión institucional reportada por los profesores en el último año.

Tabla 5. No. de ocasiones en las que el último año participaron en actividades de gestión institucional.

\begin{tabular}{|c|c|c|c|}
\hline \multirow[b]{2}{*}{ Participante } & \multicolumn{3}{|c|}{ Gestión Institucional } \\
\hline & $\begin{array}{l}\text { Diseño/Rediseño Modelo } \\
\text { Educativo y/o Programas } \\
\text { (Licenciatura y Posgrado) }\end{array}$ & $\begin{array}{l}\text { Responsabilidad, } \\
\text { Función o } \\
\text { Comisión } \\
\text { Institucional }\end{array}$ & $\begin{array}{c}\text { Nombre de las funciones } \\
\text { o Comisiones }\end{array}$ \\
\hline PTC1 Dr & 4 & 5 & $\begin{array}{l}\text {-Comisión de } \\
\text { Categorización }\end{array}$ \\
\hline PTC2 M. & 2 & 0 & -Sin función o comisión \\
\hline PTC3 Lic. & 1 & 1 & $\begin{array}{l}\text {-Comisión de Ingreso de } \\
\text { Licenciatura }\end{array}$ \\
\hline PTC4 Dr & 2 & 0 & -Sin función o comisión \\
\hline PTC5 M. & 5 & 2 & -Sin función o comisión \\
\hline PTC6 Dr & 0 & 2 & $\begin{array}{l}\text {-Comisión curricular } \\
\text {-Comisión de Admisión }\end{array}$ \\
\hline PTC7 Pos. & 5 & 0 & -Sin función o comisión \\
\hline PTC8 M. & 0 & 1 & $\begin{array}{l}\text {-Comité académico } \\
\text { COARA }\end{array}$ \\
\hline PTC9 Dr & 0 & 1 & $\begin{array}{l}\text {-Comité Académico del } \\
\text { Posgrado de la FCA }\end{array}$ \\
\hline PTC10 Dr & 14 & 6 & -Comisión Curricular \\
\hline PTC11 Dr & 0 & 0 & -Sin función o comisión \\
\hline PTC12 Dr & 1 & 0 & -Sin función o comisión \\
\hline PTC13 Dr & 1 & 2 & -Sin función o comisión \\
\hline PTC14 Dr & 2 & 0 & -Sin función o comisión \\
\hline PTC15 Dr & 1 & 1 & $\begin{array}{l}\text {-Comisión de Evaluación } \\
\text { de Beca al desempeño } \\
\text { Docente }\end{array}$ \\
\hline PTC16 Dr & 2 & 0 & $\begin{array}{l}\text {-Comisión de } \\
\text { Investigación y } \\
\text { Desarrollo Tecnológico }\end{array}$ \\
\hline PTC17 Dr & 3 & 0 & -Sin función o comisión \\
\hline TA M. & 0 & 0 & -Sin función o comisión \\
\hline PHC1 M. & 0 & 0 & -Sin función o comisión \\
\hline PHC2 M. & 0 & 0 & -Sin función o comisión \\
\hline PHC3 M. & 0 & 0 & -Sin función o comisión \\
\hline PHC4 M. & 0 & 0 & -Sin función o comisión \\
\hline PHC5 M. & 0 & 0 & -Sin función o comisión \\
\hline
\end{tabular}


Es importante señalar que solo tres profesores de los 23 participantes reportaron, que al menos en alguno de los últimos cuatro años, pertenecieron al H. Consejo Técnico de la FCA. De igual forma, del total de los participantes, nadie reportó, en este mismo periodo, haber pertenecido al H. Consejo Directivo de la UASLP.

\subsubsection{Tutorías}

Otra de las funciones que obliga a los PTC y que los TA y PHC realizan de manera voluntaria es la de tutorías. Esta función se determinó por el número de estudiantes reportados por los profesores en cada uno de los niveles educativos: licenciatura, posgrado sin o con PNPC. A continuación, se presenta la Figura 8 que muestra el total de estudiantes a los que cada uno de los profesores brinda tutorías académicas.

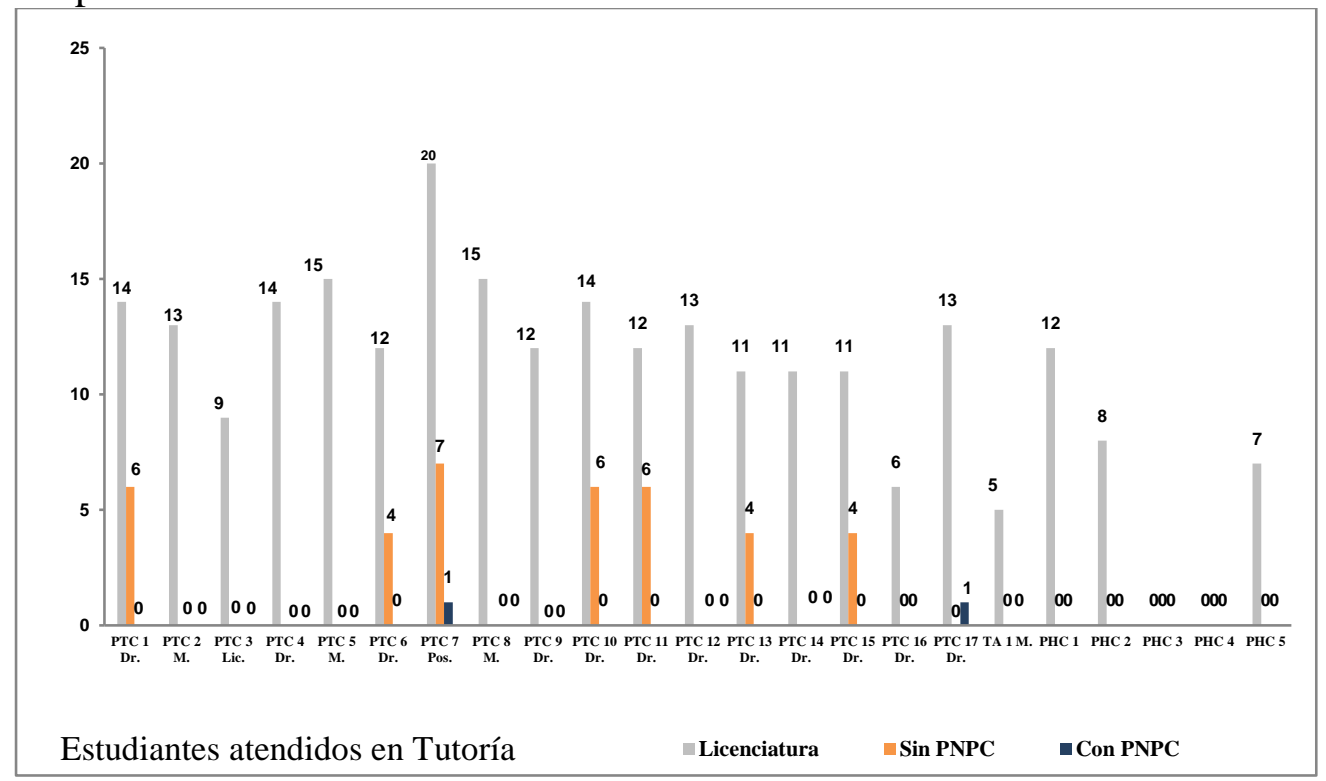

Figura 8. Total de estudiantes que reciben tutoría académica reportados por los profesores.

\section{Conclusiones}

La principal conclusión del resultado del diagnóstico fue que las actividades y funciones de los profesores participantes del estudio no están equilibradas, tomando como referencia lo establecido en el Reglamento del Personal Académico de la UASLP, las reglas de operación y convocatorias del Programa para el Desarrollo Profesional Docente del Tipo Superior (PRODEP) y el Sistema Nacional de Investigadores (SNI) del Consejo Nacional de Ciencia y Tecnología (CONACYT).

Específicamente, las actividades de la función de investigación, según los resultados, presentan las siguientes áreas de oportunidad: (a) asignar el 
mismo número de horas a la semana para investigación de los PTC, (b) balancear los apoyos financieros para el desarrollo de proyectos de investigación de todos los PTC, b) financiar a los profesores para la difusión de resultados en eventos académicos (congresos nacionales y/o internacionales) y pago de costos de publicaciones de calidad (libros, revistas indexadas), (c) la consideración equitativa para participar como director o asesor de tesis de licenciatura y posgrado.

Para el caso de la función de docencia se concluye que es necesario atender las disposiciones del Reglamento de Personal Académico que menciona el número de horas frente a grupo dependiendo del nivel académico I, II, III, IV, V y VI (UASLP, 1984).

En la función de gestión se identificó la mayor área de oportunidad porque los resultados de esta dimensión permiten concluir que la mayoría de los participantes del diagnóstico no tienen asignado desde hace dos años ninguna actividad de gestión o responsabilidad institucional permanente, solo se reportan actividades de gestión eventuales y otras que no son de la facultad.

En la función de tutorías, los profesores participantes si manifestaron participar en el Programa Institucional de Tutorías de la UASLP (seguimiento académico) sin embargo, se concluye que su participación está limitada para la titulación de tesis, actualización y de publicación de artículo a nivel licenciatura y en la opción de asesores de tesis a nivel de posgrado de preferencia en programas reconocidos por el PNPC.

\section{Recomendaciones}

A partir de los resultados obtenidos en este diagnóstico y con el propósito de coadyuvar al logro de los objetivos de investigación, docencia, tutorías y gestión establecidos en el PIDE 2013-2023 y en el PLADE 20132023 se recomienda lo siguiente:

Con respecto a la función de investigación, de acuerdo con el PRODEP y CONACYT, que los PTC dediquen un mínimo de 20 horas/semana de su carga de 40 horas/semana. Asimismo, cabe mencionar que para contribuir con los indicadores considerados en la función de Investigación es necesario anualmente: (a) mínimo dos proyectos de investigación de forma individual o colectiva, (b) contar con financiamiento interno o externo para al menos unos de los dos proyectos sugeridos, (c) al menos dirigir dos tesis de estudiantes de posgrado al año, y de preferencia en programas reconocidos por el PNPC, (c) participar en al menos dos comités de tesis de posgrado en programas no reconocidos por el PNPC y uno de licenciatura reconocida por organismos certificadores, (d) participar en al menos un evento académico al año para la difusión de resultados con el apoyo financiero de las autoridades de la facultad u organismos externos y (e) tener una participación activa como miembro o colaborador de uno de los Cuerpos Académicos que existen en la institución. 
Esta situación permitirá a los profesores tener una participación adecuada en las convocatorias de evaluación interna (UASLP/PREDO) y externas (PRODEP, CONACYT) y en consecuencia no se contribuye al logro de los indicadores de investigación, docencia, gestión y tutorías, establecidos en el PLADE 2013-2023.

Para la actividad de docencia, se recomienda considerar lo establecido en el Reglamento de Personal Académico de la UASLP, el cual establece que los PTC impartan un mínimo de hrs/sem frente a grupo de acuerdo con su nivel académico. Para el Nivel I 20 hrs/sem, Nivel II 15 hrs/sem, Nivel III 12 hrs/sem, Nivel IV nueve hrs/sem, Nivel V seis hrs/sem y Nivel VI tres hrs/sem. Sin embargo, en la Convocatoria del Programa de Estímulos al Desempeño del Personal Docente 2016-207, se estableció para participar en el programa que los PTC impartan un mínimo de cuatro hrs/sem frente a grupo. En consecuencia, se recomienda que los PTC Nivel seis impartan cuatro hrs/sem.

Por otro lado, se recomienda que las autoridades asignen las actividades de gestión necesarias para el equilibrio de funciones de los profesores. Al respecto, el Reglamento de Personal Académico de la UASLP establece que los profesores pueden desempeñar cargos de carácter directivo, administrativo o coordinación de la universidad conservando todos sus derechos académicos. Considerando que el propósito de este trabajo es describir cuantitativamente la distribución de actividades y funciones institucionales que realizan los Profesores de Tiempo Completo, Técnicos Académicos y Profesores Hora Clase de la facultad, entonces sus resultados ofrecen la oportunidad para el equilibrar de actividades y funciones de los profesores y recomendar, a partir de cada uno de los casos, el tipo de gestión institucional que se puede encomendar.

Asimismo, los profesores deben cumplir con la función de tutoría como una actividad de seguimiento académico de los estudiantes y/o asesoría de proyectos académicos. Por lo anterior se recomienda que se incluya a los profesores en el programa institucional de tutorías de la facultad, así como en el catálogo de asesores para opciones de titulación de tesis, actualización y de publicación de artículo a nivel licenciatura y en la opción de asesores de tesis a nivel de posgrado de preferencia en programas reconocidos por el PNPC.

Finalmente, es importante mencionar que contar con escenarios que permitan lograr el equilibrio de funciones y actividades de los profesores, se recomienda a mediano y largo plazo, diversificar la oferta educativa de licenciatura y posgrado. Concretamente, incrementar el número de programas educativos de licenciatura pertinentes a las necesidades de la región y como respuesta a las condiciones socioeconómicas nacionales e internacionales. Además, se recomienda incorporar a los programas de posgrado al PNPC. Lo anterior, permitirá a las autoridades de la facultad contar con más opciones 
para distribuir y equilibrar las actividades y funciones de manera más equitativa para todos los profesores de la facultad.

\section{References:}

1. CONACYT (2019). Sistema Nacional de Investigadores. https://www.conacyt.gob.mx/index.php/el-conacyt/sistema-nacionalde-investigadores

2. Figueiredo, D., Montoro, M., \& Lucena, M. (2016). La Formación Investigativa Como Salida Para El Compromiso Profesional De Futuros Profesores En Angola. European Scientific Journal, ESJ, 12(28), 18. https://doi.org/10.19044/esj.2016.v12n28p18

3. Goñi, J. (2005). El espacio europeo de educación superior, un reto para la universidad. Competencias, tareas y evaluación, los ejes del currículum universitario. Barcelona, España: OCTAEDRO- ICE-UB.

4. Hernández, R., Fernández, C. \& Baptista, P. (2014). Metodología de la investigación (6a. ed.). Distrito Federal, México: McGraw-Hill.

5. Palomares, A. (2004). Profesorado y educación para la diversidad en el siglo XXI. España: Universidad de Castilla-La Mancha.

6. Ruiz B., Mas T., Tejada J. \& Navío A. (2008). Funciones y escenarios de actuación del profesor universitario: Apuntes para la definición del perfil basado en competencias. Revista de la Educación Superior,37(146), $115-132$. http://www.scielo.org.mx/scielo.php?script=sci_arttext\&pid=S018527602008000200008

7. SEP (2019). Reglas de operación del PRODEP. http://www.dof.gob.mx/2019/SEP/ANEXO_DEL_ACUERDO_22_1 2_19.pdf

8. SEP (2019). Cuerpos Académicos reconocidos por PRODEP. http://promep.sep.gob.mx/ca1/

9. Tomàs, M. (2001). Presentación. Educar. https://educar.uab.cat/article/download/v28-tomas/358

10. UASLP (2019). Informe 2019-2020. México: UASLP.

11. UASLP (1984). Reglamento del Personal Académico de la Universidad Autónoma de San Luis Potosí. México: UASLP. 